\title{
Obésité : d'un syndrome monogénique exceptionnel aux interactions entre gènes multiples et environnement nutritionnel
}

Oléagineux, Corps Gras, Lipides. Volume 10, Numéro 2, 109-14, Mars 2003, Approche biologique

Auteur(s) : Frédéric FUMERON , EA 3516, Faculté Xavier Bichat, Université Paris 7, Denis Diderot, BP416, 75870 Paris cedex $18 \mathrm{E}$-mail : fumeronbichat.inserm.fr .

Author(s) : Frédéric FUMERON

Résumé : L'obésité a une part génétique démontrée par des études de corrélations familiales, comme les études d'adoptés ou de jumeaux élevés séparément. Une étude de jumeaux suralimentés a démontré l'importance des gènes dans la réponse à la nutrition, et donc de l'interaction gènes \nutrition. Les modèles animaux et des études métaboliques ont fourni de nombreuses pistes de gènes dits "candidats". Les gènes candidats pour l'obésité et ses complications peuvent intervenir dans la prise alimentaire, la dépense énergétique, la biologie des adipocytes, le métabolisme lipidique. Des mutations des gènes de la voie leptine-mélanocortines ont été impliquées dans les cas exceptionnels d'obésité monogénique chez l'homme. Cependant, dans le cas général, l'obésité commune est un phénotype complexe qui résulte d'interactions entre l'environnement (nutrition) et des facteurs génétiques (polygéniques) de prédisposition. Les interactions avec la nutrition peuvent être révélées par une alimentation pléthorique mais aussi par la réponse au régime restrictif, et certains polymorphismes génétiques modifient la réponse au régime hypocalorique. Puisque le séquençage du génome humain est presque terminé et va fournir une liste exhaustive des polymorphismes génétiques, les variants génétiques devront être testés dans des modèles $d$ 'interaction (études longitudinales prospectives, études d'interventions nutritionnelles). Ces études serviront à trouver de nouvelles voies physiopathologiques et thérapeutiques, plutôt qu'à estimer un risque individuel.

Summary : Familial correlations have shown the importance of the genetic background in susceptibility to obesity (by adoption studies or studies in twins reared separately). The study of identical twins submitted to overfeeding has demonstrated the importance of the genes in response to nutritional factors, i.e. the role of the interaction genes $\backslash$ nutrition. The candidate genes can be selected on the basis of rodent genetic models of obesity or of metabolic studies. This approach allowed to discover the mutations involved in the very rare mendelian obesity cases, which are linked to the leptin-melanocortin pathway. Nevertheless, common obesity is a complex phenotype resulting from interactions between environmental (mainly nutritional) and genetic (polygenic) determinants. All genes whose products have effects on energy intake, energy expenditure, lipid metabolism or adipocyte biology, can be tested as candidates. Interaction can be revealed by a plethoric diet as well as a restrictive one. Some candidate genes involved in different aspects of energy or lipid metabolism modulate the susceptibility to common obesity and associated complications, and the response to low calorie diet. The Human Genome Project has provided an (almost) exhaustive list of genetic 
polymorphisms. These genetic variants must be tested in interaction models (longitudinal prospective studies (Idietary intervention studies). These studies will help to find new physiopathologic and therapeutic ways, rather than predict an individual risk.

Mots-clés : obésité, polymorphismes génétiques, monogénique, polygénique, nutrition

Keywords : obesity, gene polymorphisms, monogenic, polygenic, nutrition

\section{ARTICLE}

\section{Auteur(s) : Frédéric FUMERON}

EA 3516, Faculté Xavier Bichat, Université Paris 7, Denis Diderot, BP416, 75870 Paris cedex 18 Email : fumeron@bichat.inserm.fr

L'obésité humaine a une composante familiale qu'on peut estimer par le coefficient $\lambda r$, rapport du risque d'être obèse si l'on possède un parent obèse sur le risque d'être obèse dans la population générale : on a 3 fois plus de "chances " d'être obèse lorsqu'on possède un parent au premier degré obèse (après exclusion des jumeaux monozygotes de la statistique !) [1]. Cependant, dans une famille, outre les gènes, on partage aussi un environnement: des habitudes alimentaires, un mode de vie plus ou moins actif II existe une interaction entre les facteurs génétiques et les facteurs d'environnement, autre-ment dit, on devient obèse si l'on possède certains gènes, pour un mode de vie donné. Quels sont les gènes responsables ? On peut étudier directement des gènes "candidats", impliqués dans la prise alimentaire, la dépense énergétique, la biologie des adipocytes, le métabolisme des lipides. D'autres études, parexamen systématique de tous les chromosomes, permettent de localiser certaines régions chromosomiques dans lesquelles des gènes connus ou inconnus prédisposent à l'obésité.

\section{Historique : l’obésité a une part génétique}

Les plus anciens arguments en faveur de la génétique dans la survenue de l'obésité humaine sont les études de ressemblances familiales. Dans ce domaine, les démonstrations probantes viennent d'études qui permettent de distinguer l'effet des gènes de celui de l'environnement, effectuées relativement récemment (années 80 ), comme les études d'adoptés ou de jumeaux élevés séparément. Au Danemark, on a démontré une forte relation entre la classe de poids de 540 sujets adoptés et l'indice de masse corporelle (IMC = poids/taille $\left.{ }^{2}\right)$ de leurs parents biologiques, aucune relation n'étant observée avec l'indice des parents adoptifs. L'environnement familial partagé semble avoir peu d'effet, en particulier après que les adoptés ont quitté le foyer familial [2]. Les études de jumeaux sont extensivement utilisées pour démontrer le caractère héréditaire de différents phénotypes. Le problème est que les vrais jumeaux partagent souvent plus le même environnement que les faux jumeaux, ou les fratries classiques. Une étude de jumeaux effectuée en Suède a montré que la corrélation (indiquant la ressemblance) intra-paire pour l'indice de corpulence était bien plus élevée chez les vrais jumeaux que chez les faux jumeaux [3]. Cette 
corrélation ne changeait pas selon que les jumeaux avaient été élevés ensemble ou séparément. Autrement dit, l'environnement ne modifiait pratiquement pas la ressemblance entre jumeaux, qui ne dépendait que du nombre de gènes en commun (100\% pour les vrais, $50 \%$ pour les faux jumeaux). Ces résultats ainsi que ceux des études d'adoption montrent que la ressemblance pour la corpulence est très liée aux gènes en commun (tableau 1).

Tableau 1. Coefficients de corrélation pour l'indice de masse corporelle chez des jumeaux élevés séparément ou ensemble (Jumeaux séparés : âge moyen à l'entrée dans l'étude $=58,6$ ans, à la séparation $=2,8$ ans ; $82 \%$ avaient été séparés avant 5 ans) [3]

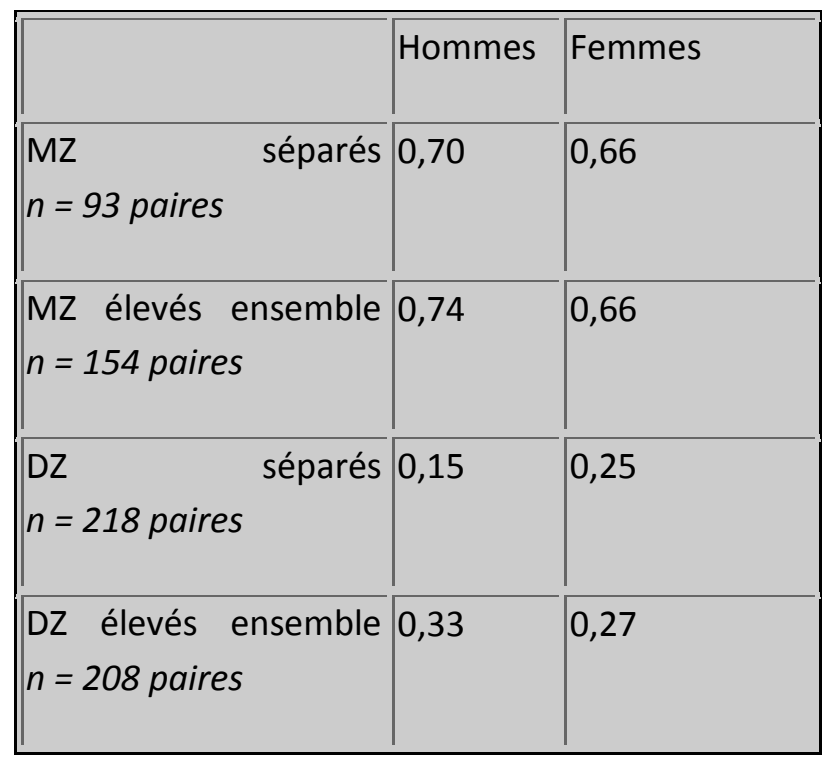

Une autre étude de jumeaux démontre l'importance des gènes dans la réponse à l'environnement nutritionnel, autrement dit de l'interaction génétique-nutrition. De vrais jumeaux (12 paires) ont été suralimentés (supplément : $1000 \mathrm{kcal} / \mathrm{jour}$ ) pendant 3 mois [4]. La ressemblance de la réponse à cette suralimentation était très élevée entre jumeaux d'une même paire, alors que d'une paire de jumeaux à une autre, cette réponse était très variable. Selon les paramètres mesurés, cette ressemblance était plus ou moins grande, montrant ainsi que les différents phénotypes de l'obésité (IMC, graisse totale, superficielle, viscérale, répartition de la graisse) n'ont pas le même déterminisme génétique. Il est intéressant de constater que la plus forte ressemblance concernait les gains dans le rapport des circonférences taille/hanches et de graisse viscérale, indices des complications métaboliques de l'obésité. Ainsi, plus encore que l'obésité simple, les complications pourraient avoir une part génétique s'exprimant en interaction avec l'environnement nutritionnel.

\section{Identification des gènes}

Les études décrites précédemment ne renseignent pas sur les gènes impliqués eux-mêmes. La biologie moléculaire permet d'étudier l'ADN directement de gènes dits " candidats " susceptibles d'avoir un effet par leur rôle métabolique ou par une mise en évidence sur des modèles animaux. Il existe des cas rares où une simple mutation entraîne l'obésité che l'homme (obésités monogéniques). Dans le cas général, les obésités sont polygéniques, ce qui explique que pris individuellement, chaque gène (ou plutôt chaque variant) aura peu d'effet, cet effet interagissant le plus souvent avec la nutrition. 
Un autre type d'étude est le criblage systématique du génome, par génotypages pour des marqueurs régulièrement espacés sur l'ensemble des chromosomes, qui a pour but de localiser certaines régions chromosomiques dans lesquelles des gènes connus ou inconnus prédisposeraient à l'obésité.

\section{Obésités monogéniques}

La cause de ces obésités réside en un défaut d'un seul gène. Ces obésités sont très sévères, avec généralement des débuts dans l'enfance. Les modèles animaux (rongeurs) sont proches de ce type d'obésité. Le premier modèle animal d'obésité génétique décrit a été la souris $o b / o b$ : hyperphage, à dépense énergétique réduite, stockant préférentiellement des calories sous forme de graisse, diabétique non insulino-dépendante. En 1994, le gène de la leptine, et sa mutation ob responsable de cette obésité, sont isolés et séquencés. La leptine est une hormone sécrétée par la cellule adipeuse et absente chez ces souris [5]. Chez les souris ob/ob, l'injection de la protéine provoque à la fois une réduction des ingesta et une augmentation des dépenses énergétiques. Chez l'homme, deux familles hautement consanguines possèdent également une obésité récessive liée à un défaut du gène de la leptine [6,7]. Les sujets atteints ont une obésité massive, avec hyperphagie, retard de développement pubertaire. Ces défauts sont corrigés par injection de leptine [8]. Les sujets hétérozygotes pour la mutation ne sont pas atteints d'obésité massive, mais ont une masse grasse accrue pour une corpulence identique ainsi qu'une leptinémie basse, comparés à des témoins non porteurs de la mutation [9].

Actuellement chez l'homme, des mutations sur 6 gènes, concernant moins de 100 individus dans le monde, ont été décrites [10] (tableau 2). Les gènes en cause sont, outre la leptine, le récepteur de la leptine (LEPR) [11], la POMC (pro-opiomélanocortine) [12], la proconvertase 1 (enzyme de maturation des hormones dont l'insuline et la POMC) [13], le récepteur hypothalamique aux mélanocortines (MC4R) [14-23], ainsi qu'un gène du développement du système nerveux, SIM1 [24]. Ces cas rares, bien que peu représentatifs des obésités communes, permettent de mieux comprendre la voie leptine-mélanocortines. La POMC est le précurseur de I'ACTH (hormone corticotrope), de la $\beta$-endorphine et de l' $\alpha$-MSH (hormone mélano-stimulante). Ce dernier peptide agit sur différents récepteurs (récepteurs de type 1-4 aux mélanocortines) pour produire des effets variés (pléiotropie) selon le type: effet anorexigène par action sur le MC4R de I'hypothalamus, mais aussi participation à la synthèse de mélanine par action sur le MC1R au niveau de la peau. En résumé, la leptine, facteur de satiété synthétisé par le tissu adipeux, agit au niveau de l'hypothalamus par un récepteur spécifique et déclenche ainsi la sécrétion d'une protéine dérivée de la POMC, $\alpha M S H$, agissant elle-même par un récepteur hypothalamique (MC4R) pour exercer un effet anorexigène (dans ce schéma, un développement normal de I'hypothalamus est nécessaire, ce qui explique l'obésité associée à une mutation de SIM1) [25].

Tableau 2. Obésités monogéniques chez l'homme [10]

Type

LEP7q31.3
Défaut primaire Phénotype

Récessif leptine

\section{Fréquence}

obésité morbide 2 familles, précoce, défaut de 6 individus puberté 


\begin{tabular}{|c|c|c|c|c|}
\hline LEPR1p31 & Récessif & récepteur de la leptine & $\begin{array}{l}\text { obésité morbide } \\
\text { précoce, défaut de } \\
\text { puberté }\end{array}$ & $\begin{array}{l}1 \text { famille, } \\
3 \text { individus }\end{array}$ \\
\hline POMC2p23.3 & Récessif & ACTH et $\alpha M S H$ & $\begin{array}{l}\text { obésité, insuffisance } \\
\text { corticotrope }\end{array}$ & $\begin{array}{l}2 \text { familles, } \\
2 \text { individus }\end{array}$ \\
\hline MC4R & Dominant & récepteur de l'aMSH & $\begin{array}{l}\text { obésité à } \\
\text { phénotype variable }\end{array}$ & $\begin{array}{l}29 \text { variants } \\
73 \text { individu }\end{array}$ \\
\hline $18 q 22$ & & & & \\
\hline $\begin{array}{l}\text { Proconvertase } \\
\text { (PCSK1)5q15-q2 }\end{array}$ & I Récessif & 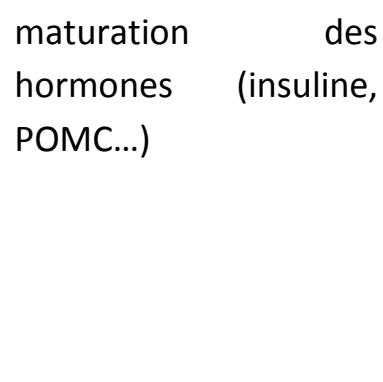 & $\begin{array}{l}\text { obésité, insuffisance } \\
\text { corticotrope } \\
\text { modérée, } \\
\text { aménorrhée, } \\
\text { troubles } \\
\text { métabolisme } \\
\text { glucidique }\end{array}$ & $\begin{array}{l}1 \text { famille, } \\
1 \text { individu }\end{array}$ \\
\hline SIM1 & Dominant & $\begin{array}{l}\text { développement } \\
\text { l'hypothalamus }\end{array}$ & $\begin{array}{l}\text { obésité } \\
\text { précoce }\end{array}$ & $\begin{array}{l}1 \text { famille, } \\
1 \text { individu }\end{array}$ \\
\hline
\end{tabular}

6q16.3-q21

Le déficit en MC4R semble être la cause d'obésité monogénique la plus fréquente, jusqu'à $6 \%$ des obésités infantiles selon les populations étudiées. Fin 2002, 29 mutations avaient été décrites chez 73 individus [10, 14-23]. De plus, c'est la seule forme monogénique chez l'homme qui soit dominante puisque la plupart des sujets porteurs sont hétérozygotes pour la mutation. Cependant, chez certains individus porteurs, la preuve que la mutation du gène est la cause de l'obésité n'est pas faite. Dans une série anglaise de 500 sujets atteints d'une obésité sévère ayant débuté dans l'enfance, 29 sujets $(5,8 \%)$ sont porteurs de mutations (24 variants différents) du MC4R, dont 6 homozygotes seulement [26]. Chez ces derniers, l'obésité est cependant plus sévère, et on peut remarquer que dans les familles, certains individus hétérozygotes ne sont pas atteints, ce qui est l'indice d'interactions avec l'environnement, ou avec d'autres gènes. L'obésité chez les porteurs de mutations du MC4R n'est donc pas toujours strictement monogénique. Dans cette dernière série bien caractérisée, le phénotype inclut une hyperphagie. Une étude suisse publiée dans le même prestigieux journal que la précédente montre que tous les obèses porteurs de mutations du MC4R $(n=24)$ ont des accès de boulimie, présente chez $14 \%$ seulement des 445 obèses témoins, non porteurs [27].

\section{Études de gènes candidats et exemples d'interactions gènes/nutrition dans les obésités communes}

L'obésité résulte d'un excès d'apports énergétiques (ingesta) par rapport aux dépenses. Les candidats peuvent donc être impliqués dans la prise alimentaire (signaux ou neuromédiateurs orexigènes ou anorexigènes : leptine et récepteur, neuropeptide $Y$, récepteurs et transporteur de la sérotonine, etc.), la dépense énergétique (protéines découplantes, leptine, récepteurs aux 
catécholamines, etc.), la biologie des adipocytes (facteurs de différenciation adipocytaire comme PPAR $\gamma$, cytokines) [10]. Les complications de l'obésité peuvent elles aussi être modulées par des facteurs génétiques, en particulier les dyslipoprotéinémies et le diabète de type 2 (gènes du métabolisme des lipides ou du glucose : apolipoprotéines, récepteurs, transporteurs, etc.).

Chaque année, la revue Obesity Research fait le point sur les études des gènes dans l'obésité [10]. Actuellement, dans la susceptibilité à l'obésité commune, multifactorielle et polygénique, plus de 300 gènes ont été décrits dont les variations ont un impact sur l'obésité et/ou ses complications dans des études d'association (populations de sujets non apparentés, études castémoins) ou de liaison (études familiales : transmission conjointe du phénotype et du marqueur génétique étudié). Ces variations sont de type "polymorphismes", définis par une fréquence des variants rares supérieure à $1 \%$ dans la population. Les polymorphismes peuvent être fonctionnels (provoquant une modification de la séquence protéique, d'un site de transcription ou d'un site important pour la stabilité du mRNA) ou non (changements d'une base dans un intron, polymorphismes de répétition dans des régions " neutres » du génome = microsatellites). Dans ce cas, un résultat significatif peut-être indirect, dû à un déséquilibre de liaison avec une mutation ou un variant fonctionnel du gène encore non connu.

Les effets des polymorphismes ne sont pas toujours retrouvés d'une étude à l'autre. Les causes de discordances peuvent être des résultats faussement positifs (ou faussement négatifs) par biais ou manque de puissance statistique, mais aussi en raison d'interactions avec l'environnement, la nutrition en particulier. Ces interactions s'expriment face à un environnement "occidental » (accès facile à une alimentation de haut densité énergétique, activité physique restreinte...), mais on peut les mettre en évidence également en réponse à des restrictions (régime hypocalorique chez les sujets en surpoids). Les exemples qui suivent sont largement tirés de nos études au Laboratoire de Nutrition Humaine de la faculté Xavier Bichat.

\section{Protéine découplante du tissu adipeux brun : UCP1}

Le tissu adipeux brun est spécialisé dans la dissipation d'énergie par l'intermédiaire d'une protéine, la protéine découplante (UCP1). Chez les rongeurs, la destruction ou une diminution d'activité du tissu adipeux brun entraînent l'obésité. Chez l'homme, il existe des dépôts de tissu adipeux brun seulement chez les nourrissons. On a pu cependant détecter une production d'UCP1 chez l'adulte sain, indiquant la présence de cellules adipeuses brunes disséminées. Un variant du gène de I'UCP1 prédispose à un gain accru de masse grasse au cours du temps (Québec Family Study: suivi longitudinal de 12 ans) [28]. Le même variant est associé à une résistance à un régime hypocalorique modéré (diminution de $25 \%$ des apports énergétiques pendant 2-3 mois) : la perte de poids varie de 4,6 kg à 7,1 kg selon le génotype [29]. Ces résultats indiquent que des cellules adipeuses brunes pourraient jouer un rôle dans l'équilibre pondéral, même chez l'homme adulte. On a découvert maintenant d'autres protéines découplantes (UCP2 ubiquitaire, UCP3 musculaire) dont les variations génétiques sont associées à des variations de la dépense énergétique et à une susceptibilité au surpoids [10].

\section{La leptine}

Le déficit total en leptine n'existe que dans deux familles, et la leptine étant produite par le tissu adipeux, les obèses sont pour la plupart hyperleptinémiques. Cependant, des polymorphismes 
du gène de la leptine, n'entraînant pas de déficit, ont été découverts [30]. Un de ces polymorphismes, G-2548A, a été relié à l'obésité commune multifactorielle aux USA [31] et en France [32], et à une réponse variable au régime hypocalorique : les sujets perdent plus ou moins de poids selon leur génotype [30]. La relation entre masse adipeuse et concentration de leptine est également modulée par ce polymorphisme [33]. Une étude récente montre que ce polymorphisme aurait un caractère fonctionnel en modifiant l'expression du gène au niveau transcriptionnel [34]. Certains polymorphismes du gène du récepteur de la leptine donnent des résultats similaires : susceptibilité au surpoids et modulation de la réponse au régime restrictif [35].

Le PPARY (peroxisome proliferator activated receptor $\gamma$ ) est un facteur de transcription, indispensable à la différenciation du tissu adipeux. Les acides gras se lient à ce récepteur avec des affinités différentes en fonction de la longueur de la chaîne et de leur insaturation. Deux formes de la protéine existent, différentes seulement par un acide aminé (Pro ou Ala) [36]. Des résultats divergents sur l'effet de cette variation dans la susceptibilité à l'obésité ont été publiés, qui pourraient être dus à une interaction. Quand le rapport des acides gras polyinsaturés/saturés $(P / S)$ de l'alimentation est bas, I'IMC et l'insuline à jeun sont augmentés chez les porteurs du variant Ala. Inversement, quand le rapport $\mathrm{P} / \mathrm{S}$ est élevé $(>0,66)$, les sujets porteurs du génotype commun Pro/Pro sont les plus corpulents, avec une insulinémie plus élevée [37].

Les complications de l'obésité sont influencées également par des facteurs génétiques. Tous les obèses ne sont pas hyperlipidémiques ou diabétiques de type 2. De nombreux gènes du métabolisme des lipides (apolipoprotéine E [38], lipoprotéine lipase [LPL] [39], etc.) modifient les effets de l'obésité sur ceux-ci. Encore une fois, des interactions existent. La LPL est l'enzyme-clé de la lypolyse circulante des lipoprotéines riches en triglycérides. Plusieurs polymorphismes influencent les concentrations des lipoprotéines plasmatiques. L'un d'entre eux, le polymorphisme HindIII du gène de la LPL est associé à des variations du bilan lipidique après un régime hypocalorique [40], ou après une suralimentation (dans l'expérience classique des jumeaux) [41] (tableau 3). Dans la prédisposition au diabète de type 2 chez les obèses, on peut mentionner le polymorphisme de PPARy [36], ainsi que ceux d'une protéine récemment découverte, l'adiponectine, produite par le tissu adipeux, ayant une action insulinosensibilisatrice due à une augmentation de l'oxydation, lipidique en particulier [42-47].

Tableau 3. Triglycérides ( $\mathrm{mmol} / \mathrm{I})$ en réponse à un régime hypocalorique ou à une suralimentation en fonction du génotype hindiii de la LPL

\begin{tabular}{lrrrrr} 
& avant & après & \multicolumn{2}{c}{ avant } & \\
& & & & \\
obèses au régime hypocalorique $[40]$ & 1,07 & 1,03 & 1,39 & 1,12 \\
jumeaux suralimentés [41] & 1,00 & 1,32 & 1,31 & 2,26
\end{tabular}

L'enzyme de restriction HindIIIdétermine un polymorphisme à deux allèles sur le gène de la LPL (présence - $\mathrm{H2}$ - ou absence - $\mathrm{H} 1$ - d'un site de coupure de l'ADN par changement d'une seule base nucléotidique). Les sujets porteurs du génotype $\mathrm{H} 2 \mathrm{H} 2$ ont une concentration de triglycérides plus élevée, chez les obèses et chez les jumeaux (minces) en régime spontané. Ils sont les plus 
sensibles à une variation des apports énergétiques, soit sous forme d'une restriction, soit d'une suralimentation.

\section{Genome scan}

Le criblage du génome ("genome scan ") ne concerne pas directement des gènes mais des régions chromosomiques. Le principe consiste à étudier dans des familles la transmission d'un trait phénotypique, qualitatif ou quantitatif (= QTL dans ce cas) avec des marqueurs génétiques dont on connaît la localisation (mais pas le rôle) tout le long du génome. Ces techniques sont particulièrement coûteuses, puisque des centaines de marqueurs différents doivent être étudiés sur des centaines de sujets au moins. La plus important étude de ce type, la NHLBIFHS aux USA [48], sur deux échantillons, un de 1184 sujets et l'autre de 3027 , montre deux régions fortement liées, $7 q 32$ proche du gène de la leptine expliquant $28 \%$ de la variation de l'IMC $(p<0,00001$ !) et 13q14, proche du gène du récepteur de la sérotonine 5HT2R dont des polymorphismes ont été associés au comportement alimentaire et à la consommation énergétique [49]. Actuellement, on dispose de résultats sur une vingtaine de populations environ $[10,50]: 18$ régions chromosomiques ont été répliquées au moins deux fois, et 5 au moins trois fois. Parmi ces dernières, l'une est particulièrement intéressante, 3q27, liée au syndrome métabolique (obésité, diabète de type 2, athérome) et contenant le gène de l'adiponectine [51, 52]. Cependant, tous les résultats obtenus par le criblage du génome doivent être affinés pour trouver le ou les gènes importants, puisqu'une région impliquée peut recouvrir une centaine de gènes différents.

\section{Conclusion}

La recherche génétique a grandement contribué à l'amélioration récente des connaissances sur l'obésité de ces dernières années. Son apport est considérable dans le domaine de la physiologie et de la pathophysiologie. Le rôle du tissu adipeux a ainsi été réévalué par la découverte des adipocytokines comme la leptine, hormone qui joue sur la prise alimentaire, la dépense énergétique, le maintien de l'équilibre pondéral, mais aussi le développement pubertaire, l'angiogénèse, le développement osseux... II est vraisemblable que l'adiponectine démontrera aussi tout un ensemble d'effets pléiotropiques.

Les études génétiques ont permis paradoxalement de réévaluer le rôle de l'environnement, puisque la variabilité génétique influence la réponse à l'environnement, en particulier la nutrition, pour moduler la prédisposition à l'obésité. Puisque le séquençage du génome humain est presque terminé et va fournir une liste exhaustive des polymorphismes génétiques [53], les variants génétiques devront être testés dans des modèles d'interaction (études longitudinales prospectives, études d'interventions nutritionnelles).

Les études génétiques ne permettent pas d'évaluer un risque individuel. Actuellement, plus de 300 gènes ou régions chromosomiques de prédisposition sont connus et leur nombre augmente tous les jours. La prédisposition résulte des interactions entre ces gènes (et d'autres, encore inconnus à ce jour), ainsi qu'entre gènes et environnement. Autrement dit, l'évaluation d'un risque individuel dépend d'une batterie de tests génétiques dont l'emploi en routine n'est pas pour demain. En revanche, la génétique pourra peut-être permettre une meilleure évaluation des risques de succès ou d'échec des traitements futurs, médicamenteux et/ou nutritionnels (pharmacogénomique). 


\section{RÉFÉRENCES}

1. Allison DB, Faith MS, Nathan JS. Risch's lambda values for human obesity. Int J Obes Relat Metab Disord $1996 ; 20$ : 990-9.

2. STUNKARD AJ, SORENSEN TIA, HANIS C, et al. An adoption study of human obesity. N Engl J Med $1986 ; 314: 193-8$.

3. STUNKARD AJ, HARRIS JR, PEDERSEN NL, MAC CLEARN GE. The body mass index of twins who have been reared apart. N Engl J Med $1990 ; 322$ : 1483-7.

4. Bouchard C, Tremblay A, Després JP, et al. The response to long term overfeeding in identical twins. N Engl J Med $1990 ; 322: 1477-82$.

5. Zhang Y, Proenca R, Maffei M, Barone M, Leopold L, Friedman JM. Positional cloning of the mouse obese gene and its human homologue. Nature $1994 ; 372: 425-32$.

6. Montague CT, Farooqi IS, Whitehead JP, et al. Congenital leptin deficiency is associated with severe early-onset obesity in humans. Nature $1997 ; 387$ : 903-8.

7. Strobel A, Issad T, Camoin L, Ozata M, Strosberg AD. A leptin missense mutation associated with hypogonadism and morbid obesity. Nat Genet $1998 ; 18: 213-5$.

8. Farooqi IS, Jebb SA, Langmack $G$, et al. Effects of recombinant leptin therapy in a child with congenital leptin deficiency. N Engl J Med 1999 ; 34 : 879-84.

9. Farooqi IS, Keogh JM, Kamath $S$, et al. Partial leptin deficiency and human adiposity. Nature $2001 ; 414: 34-5$.

10. Chagnon YC, Rankinen T, Snyder EE, Weisnagel SJ, Pérusse L, Bouchard C. The human obesity gene map : the 2002 update. Obes Res $2003 ; 11$ : 313-67.

11. Clément $\mathrm{K}$, Vaisse $\mathrm{C}$, Lahlou $\mathrm{N}$, et al. A mutation in the human leptin receptor gene causes obesity and pituitary dysfunction. Nature $1998 ; 392$ : 398-401.

12. Krude H, Biebermann H, Luck W, Horn R, Brabant G, Gruters A. Severe early-onset obesity, adrenal insufficiency and red hair pigmentation caused by POMC mutations in humans. Nat Genet $1998 ; 19: 155-7$.

13. Jackson RS, Creemers JWM, Ohagi S, et al. Obesity and impaired prohormone processing associated with mutations in the human prohormone convertase 1 gene. Nat Genet $1997 ; 16$ : 303-6.

14. Ozata M, Ozdemir IC, Licinio J. Human leptin deficiency caused by a missense mutation : multiple endocrine defects, decreased sympathetic tone, and immune system dysfunction indicate new targets for leptin action, greater central than peripheral resistance to the effects of leptin, and spontaneous correction of leptin-mediated defects. J Clin Endocrinol Metab 1999 ; $84: 3686-95$. 
15. Hinney A, Schmidt A, Nottebom K, et al. Several mutations in the melanocortin-4 receptor gene including a nonsense and a frameshift mutation associated with dominantly inherited obesity in humans. J Clin Endocrinol Metab 1999 ; 84 : 1483-6.

16. Yeo GSH, Farooqi IS, Aminian S, Halsall DJ, Stanhope RG. A frameshift mutation in MC4R associated with dominantly inherited human obesity. Nat Genet $1998 ; 20$ : 111-2.

17. Sina $M$, Hinney A, Ziegler A, et al. Phenotypes in three pedigrees with autosomal dominant obesity caused by haploinsufficiency mutations in the melanocortin-4 receptor gene. Am J Hum Genet $1999 ; 65: 1501-7$.

18. Vaisse C, Clement K, Guy-Grand B, Froguel P. A frameshift mutation in human MC4R is associated with a dominant form of obesity. Nat Genet $1998 ; 20: 113-4$.

19. Vaisse C, Clement K, Durand E, Hercberg S, Guy-Grand B, Froguel P. Melanocortin-4 receptor mutations are a frequent and heterogeneous cause of morbid obesity. J Clin Invest $2000 ; 106$ : $253-62$.

20. Gu W, Tu Z, Kleyn PW, et al. Identification and functional analysis of novel human melanocortin-4 receptor variants. Diabetes $1999 ; 48: 635-9$.

21. Farooqi IS, Yeo GS, Keogh JM, et al. Dominant and recessive inheritance of morbid obesity associated with melanocortin receptor deficiency. J Clin Invest $2000 ; 106$ : 271-9.

22. Mergen $M$, Mergen $H$, Ozata $M$, Oner R, Oner C. A novel melanocortin 4 receptor (MC4R) gene mutation associated with morbid obesity. J Clin Endocrinol Metab 2001 ; 86 : 3448-51.

23. Dubern B, Clement K, Pelloux V, et al. Mutational analysis of melanocortin- 4 receptor, agoutirelated protein, and alphamelanocyte-stimulating hormone genes in severely obese children. J Pediatr $2001 ; 139$ : 204-9.

24. Holder JL Jr, Butte NF, Zinn AR. Profound obesity associated with a balanced translocation that disrupts the SIM1 gene. Hum Mol Genet $2000 ; 9$ : 101-8.

25. Cummings DE, Schwartz MW. Genetics and pathophysiology of human obesity Annu Rev Med $2003 ; 54: 453-71$.

26. Farooqi IS, Keogh JM, Yeo GSH, Lank EJ, Cheetham T, O'Rahilly S. Clinical spectrum of obesity and mutations in the melanocortin 4 receptor gene. N Engl J Med 2003 ; 348 : 1085-95.

27. Branson R, Potoczna N, Kral JG, Lentes KU, Hoehe MR, Horber FF. Binge eating as a major phenotype of melanocortin 4 receptor gene mutations. N Engl J Med 2003 ; 348 : 1096-103.

28. Oppert JM, Vohl MC, Chagnon $M$, et al. DNA polymorphism in the uncoupling protein (UCP) gene and human body fat. Int J Obes Relat Metab Disord 1994 ; 18 : 526-31.

29. Fumeron F, Durack-Bown I, Betoulle DC, et al. Polymorphisms of uncoupling protein (UCP) and $\beta 3$ adrenoreceptor genes in obese people submitted to a low calorie diet. Int J Obes Relat Metab Disord 1996 ; 20 : 1051-4. 
30. Mammès $O$, Betoulle $D$, Aubert $R$, et al. Novel polymorphisms in the $5^{\prime}$ region of the LEP gene : Association with leptin levels and response to low calorie diet in human obesity. Diabetes $1998 ; 47: 487-9$.

31. Li WD, Reed DR, Lee JH, et al. Sequence variants in the $5^{\prime}$ flanking region of the leptin gene are associated with obesity in women. Ann Hum Genet $1999 ; 63$ : 227-34.

32. Mammès $O$, Betoulle $D$, Aubert $R$, Herbeth $B$, Siest $G$, Fumeron $F$. Association of the G-2548A polymorphism in the $5^{\prime}$ region of the LEP gene with overweight. Ann Hum Genet $2000 ; 64$ : 391 94.

33. Le Stunff C, Le Bihan C, Schork NJ, Bougnères P. Common promoter variant of the leptin gene is associated with changes in the relationship between serum leptin and fat mass in obese girls. Diabetes $2000 ; 49: 2196-200$.

34. Hoffstedt J, Eriksson P, Mottagui-Tabar S, Arner P. A polymorphism in the leptin promoter region (-2548 G/A) influences gene expression and adipose tissue secretion of leptin. Horm Metab Res $2002 ; 34: 355-9$.

35. Mammès $O$, Aubert $R$, Betoulle $D$, et al. LEPR gene polymorphisms : associations with overweight, fat mass and response to diet in women. Eur J Clin Invest 2001 ; 31 : 398-404.

36. Stumvoll $M$, Häring $H$. The peroxisome proliferator - activated receptor- $\gamma 2$ Pro12Ala polymorphism. Diabetes $2002 ; 51: 2341-7$.

37. Luan J, Browne PO, Harding AH, et al. Evidence for gene-nutrient interaction at the PPARY locus. Diabetes $2001 ; 50: 686-9$.

38. Fumeron F, Rigaud D, Bertière MC, Bardon S, Dely C, Apfelbaum M. Association of apolipoprotein 4 allele with hypertriglyceridemia in obesity. Clin Genet $1988 ; 34: 258-64$.

39. Jemaa R, Tuzet S, Portos $C$, Betoulle D, Apfelbaum M, Fumeron F. Lipoprotein lipase gene polymorphisms : association with hypertriglyceridemia and body mass index in obese people. Int J Obes Relat Metab Disord $1995 ; 19: 270-4$.

40. Jemaa R, Tuzet $S$, Betoulle $D$, Apfelbaum M, Fumeron F. Hind III polymorphism of the lipoprotein lipase gene and plasma lipid response to low calorie diet. Int J Obes Relat Metab Disord $1997 ; 21: 280-3$.

41. Ukkola O, Tremblay A, Bouchard C. Lipoprotein lipase polymorphisms and responses to longterm overfeeding. J Intern Med $2002 ; 251: 429-36$.

42. Hara K, Boutin $\mathrm{P}$, Mori Y, et al. Genetic variation in the gene encoding adiponectin is associated with an increased risk of type 2 diabetes in the Japanese population. Diabetes 2002 ; $51: 536-40$.

43. Stumvoll M, Tschritter O, Fritsche A, et al. Association of the T-G polymorphism in adiponectin (exon 2 ) with obesity and insulin sensitivity : interaction with family history of type 2 diabetes. Diabetes $2002 ; 51$ : 37-41. 
44. Menzaghi C, Ercolino T, Di Paola R, et al. A haplotype at the adiponectin locus is associated with obesity and other features of the insulin resistance syndrome. Diabetes $2002 ; 51: 2306-$ 12 .

45. Kondo $\mathrm{H}$, Shimomura I, Matsukawa $\mathrm{Y}$, et al. Association of adiponectin mutation with type 2 diabetes : A candidate gene for the insulin resistance syndrome. Diabetes $2002 ; 51: 2325-8$.

46. Vasseur F, Helbecque N, Dina C, et al. Single-nucleotide polymorphism haplotypes in the both proximal promoter and exon 3 of the APM1 gene modulate adipocyte-secreted adiponectin hormone levels and contribute to the genetic risk for type 2 diabetes in French Caucasians. Hum Mol Genet $2002 ; 11: 2607-14$.

47. Fumeron $F$, Betoulle $D$, Péan $F$, et al. Adiponectine : effets indépendants du polymorphisme $\mathrm{T} 45 \rightarrow \mathrm{G}$ et de sa concentration sur le risque d'hyperglycémie à 3 ans dans l'étude DESIR. Diabetes Metab $2003 ; 29$ : 1 S34.

48. Feitosa ML, Borecki IB, Rich SS, et al. Quantitative-trait loci influencing body-mass index reside on chromosomes 7 and 13 : The National Heart, Lung, and Blood Institute Family Heart Study. Am J Hum Genet $2002 ; 70$ : 72-82.

49. Aubert R, Betoulle $D$, Herbeth $B$, Siest G, Fumeron F. 5-HT2A receptor gene polymorphism is associated with food and alcohol intake in obese people. Int J Obes Relat Metab Disord 2000 ; 24 : 920-4.

50. Commuzzie AG. The emerging pattern of the genetic contribution to human obesity. Best Pract Res Clin Endocrinol Metab $2002 ; 16$ : 611-21.

51. Kissebah AH, Sonnenberg GE, Myklebust J, et al. Quantitative trait loci on chromosomes 3 and 17 influence phenotypes of the metabolic syndrome. Proc Natl Acad Sci USA $2000 ; 97$ : 14478-83.

52. Vionnet N, Hani EH, Dupont S, et al. Genomewide search for type 2 diabetes-susceptibility genes in French whites : Evidence for a novel susceptibility locus for early-onset diabetes on chromosome 3q27-qter and independent replication of a type 2-diabetes locus on chromosome 1q21-q24. Am J Hum Genet $2000 ; 67: 1470-80$.

53. The international SNP map working group. A map of human genome sequence variation containing 1.42 million single nucleotide polymorphisms. Nature $2001 ; 409$ : 928-33.

$\mathrm{h} 2 \mathrm{~h} 2$ 
